\title{
SupraNova Events from Spun-up Neutron Stars: an Explosion in Search of an Observation
}

\author{
Mario Vietri ${ }^{1}$ and Luigi Stella ${ }^{2}$ \\ ${ }^{1}$ Università di Roma 3, Via della Vasca Navale 84, 00147 Roma, \\ Italy, E-mail: vietri@corelli.fis.uniroma3.it \\ 2 Osservatorio Astronomico di Roma, Via Frascati 33, 00040 Monteporzio Catone (Roma) \\ Italy, E-mail: stella@heads.mporzio.astro.it \\ Affiliated to I.C.R.A.
}

Received __; accepted _ _ 


\begin{abstract}
We consider a formation scenario for supramassive neutron stars (SMNSs) taking place through mass and angular momentum transfer from a close companion during a Low Mass X-ray Binary (LMXB) phase, with the ensuing suppression of the magnetic field. We show that this formation channel is likely to work for all equations of state except the stiffest ones, $\mathrm{L}, \mathrm{N}^{\star}$. After the end of the mass transfer phase, SMNSs will loose through magnetic dipole radiation most of their angular momentum, triggering the star's collapse to a black hole. We discuss the rate of occurrence of these collapses, and propose that these stars, because of the baryon-clear environment in which the implosion/explosion takes place, are the originators of gamma ray bursts.
\end{abstract}

Subject headings: gamma rays: bursts - stars: neutron - black holes - relativity: general - instabilities

\title{
1. Introduction
}

One of the key requests on gamma ray burst (GRB) models is that they make contact with the fireball model (Rees and Mèszàros 1992) which has proven so successful in predicting and interpreting the observed properties of GRBs' afterglows. In particular, this entails that a large explosion is to take place in a region with small baryon contamination: for $E=10^{53} \mathrm{erg}$, the baryon contamination must be at most $E / \gamma c^{2} \approx 10^{-4} \mathrm{M}_{\odot}$, for $\gamma=300$, the bulk Lorenz factor of the explosion. Vietri and Stella (1998) presented a model which could accomplish this, involving a supramassive neutron star (SMNS), i.e., a neutron star with a larger baryon number than any normal neutron star because it derives part of its support against self-gravity from the centrifugal force; these supramassive stars cannot 
be slowed down to zero spin rate, because they are so massive that, as they lose angular momentum, they become unstable to black hole formation before reaching zero spin rate (Cook, Shapiro and Teukolsky 1994a,b, Salgado et al., 1994). The model consists of the implosion/explosion (I/E) of a supramassive neutron star which has lost through magnetic dipole radiation so much angular momentum that it must then collapse to a black hole; the rotational energy of the small amount of equatorial mass left behind because already in near centrifugal equilibrium provides the energy source that powers the burst.

In paper I we proposed that SMNSs are formed in the SN explosion of a core with too much mass and angular momentum to end up in a normal neutron star. Though still nothing stands against this possibility, and we are not reneging it, we have now realized that a different channel exists: mass and angular momentum accretion from a companion in a Low Mass X-ray Binary (LMXB). The following discussion is relevant to the formation of MilliSecond Pulsars (MSPs), and will mimick arguments used in discussing the evolution of LMXBs into MSPs. We first discuss how the accretion of large amounts of mass and angular momentum may be realized in Nature and then apply this scenario to GRBs.

\section{Mass and angular momentum accretion}

The main obstacle to accretion onto a normal neutron star of large amounts of angular momentum from a close companion via an accretion disk is the neutron star's magnetic field: the neutron star can rotate only so fast as to make the corotation and Alfvèn radii coincide, lest a propeller phase sets in, which would actually entail angular momentum loss (Ghosh and Lamb 1978, Illarionov and Sunyaev 1975). The coincidence of these two radii

leads to an equilibrium period, $P_{e q}=1.3\left(B / 10^{12} G\right)^{6 / 7}\left(\dot{M} / \dot{M}_{E d d}\right)^{-3 / 7} \mathrm{~s}$ (Ghosh and Lamb 1992), which clearly shows that $B$ must decrease before significant spin-up can occur. Though no unique model has emerged yet, the current consensus is that the neutron star 
magnetic field decays by at least $3-4$ decades either as a direct result of mass accretion (Phinney and Kulkarni 1994) or of the ensuing spinup (Ruderman, Zhu and Chen 1998).

The strongest constraints on field decay in NSs come from LMXBs and MSPs. In only one LMXB, SAX J1808.4-3658 a coherent $2.5 \mathrm{~ms}$ signal has been detected in the persistent X-ray emission, providing direct evidence for the presence of a small magnetosphere; the inferred magnetic field is in the $B \sim 10^{8}-10^{9} \mathrm{G}$ range (Psaltis and Chakrabarty 1998). All other LMXBs have undetectably small coherent pulsations in their persistent emission, if at all. Yet spin periods in the in the 2-4 ms range have been deduced for about ten LMXBs from the X-ray flux oscillations that are present during type I bursts emitted by these sources (cf. van der Klis 1998). Spinup through accretion can have occurred in these neutron stars only if their magnetic field is lower than $\sim 10^{9} \mathrm{G}$. Further evidence that the field might have decayed to dynamical insignificance derives from the modelling of the $\mathrm{kHz}$ Quasi Periodic Oscillations (QPO), a common phenomenon observed in LMXBs. In the sonic point model (Miller, Lamb and Psaltis 1998), the Alfvén radius is located at a radius corresponding to a Keplerian frequency of $\approx 350 \mathrm{~Hz}$, corresponding to a magnetic field of $\approx 8 \times 10^{8} \mathrm{G}$ : this already bears witness to a thousand-fold reduction of the magnetic field below that of a typical newborn pulsar. A better model explains QPOs in terms of the fundamental frequencies of test particle motions in the general-relativistic potential well in the vicinity of the neutron star (Stella and Vietri 1999). The model is capable of explaining the observed relation between peak QPO frequency and its lower frequency counterpart over three orders of magnitude in peak QPO frequency and several distinct classes of sources, including candidate black holes and LMXBs (Stella, Vietri and Morsink 1999), implying that the magnetic field has been reduced already to $\lesssim 2 \times 10^{8} \mathrm{G}$ during the LMXB phase.

A different argument involves a handful of MSPs with observed magnetic fields $\lesssim 2 \times 10^{8} \mathrm{G}$, of which there are currently about a dozen, including the lowest fields ever 
measured, $7 \times 10^{7} G$ in $J 2229+2643$ and J2317 + 1439 (Camilo, Nice and Taylor 1996). For these small fields, the Alfvén radius for disk accretion (Ghosh and Lamb 1978, 1992) is smaller than $\sim 12 \mathrm{~km}$, which, according to Cook, Shapiro and Teukolsky (1994b) is larger than the radius of the innermost stable orbit for the softest equations of state for a neutron star with $M=1.4 \mathrm{M}_{\odot}$ (see Table 1 ). It should also be noticed that it has been argued cogently (Arons 1993) that the magnetic field in these objects is dominated by the dipole component, with negligible contribution from higher multipoles. Altogether, this means that we have already observed the result of accretion from a companion pushing the magnetic field to dynamical irrelevance (or, at least, very close to it) sometime during the mass exchange process. The argument about QPOs (Stella and Vietri 1999) implies that this may happen reasonably early in the LMXB history.

Detailed models are required to establish the exact history of a neutron star's mass, angular momentum and magnetic field, but unfortunately these computations are currently fraught with uncertainties: where is the $B$ field located, in the core or in the crust? And what is an appropriate model for the field suffocation? Population synthetic studies of this phenomenon (Possenti et al., 1999) have focused on two representative equations of state, and modeled the decay of the magnetic field in two limiting cases, imposing at the crust-core boundary either complete field expulsion by the superconducting core, or advection and freezing in a very highly conducting transition shell. The main result lies in the establishment of the existence of a tail in the rotation period distribution extending well beyond the shortest period observed so far $(P=1.558 \mathrm{~ms})$, with only moderate dependence on the field suppression mechanism. For the softest equation of state the period distribution is still increasing at the shortest value before the onset of mass shedding, where Possenti et al. stopped their computations, while for the stiffest one the period distribution had a wide maximum around $P=2-4 \mathrm{~ms}$, and a tail extending below this value. The fraction of objects with $P<1.558 \mathrm{~ms}$ is $\approx 1 \%$ and $\approx 10 \%$ for the stiff and soft equation of state, 
respectively, all ending up with very small magnetic fields, $\lesssim 10^{8} \mathrm{G}$.

Though the accreted mass is larger when account is taken of the need to suppress the magnetic field than when the magnetic field is neglected, the difference is not very large (Burderi et al., 1999). So, in order to appraise whether the neutron stars thusly formed may be (or not) supramassive, we simply consider Table I, from Cook, Shapiro and Teukolsky (1994b). It shows that the total amount of mass that needs being accreted from a companion in order to reach the supramassive stage at the initial point of mass shedding depends strongly upon the equation of state. For equation of state $\mathrm{C}$, the neutron star collapses to a black hole even before reaching mass shedding. The soft equations of state, EoSs, (A, D, E, KC) have become supramassive; the intermediate EoSs (C, M, UT, FPS) are within $<0.1 \mathrm{M}_{\odot}$ of doing so, and will cross the threshold if accretion continues after the mass shedding point is reached (see below). The total amounts of mass required to become supramassive $(\approx 4 / 3$ of the difference in mass at infinity, Phinney and Kulkarni 1994 , corresponding to a further $\left.\approx 0.5 \mathrm{M}_{\odot}\right)$ are so modest that it seems likely that even the models based upon EoSs AU and UU will reach this stage, provided a donor of sufficient mass is found: this too will be discussed below. EoSs L and $\mathrm{N}^{\star}$ are hopeless: the total amount of mass to be accreted corresponds to $\approx 2 \mathrm{M}_{\odot}$. If either of these EoSs were correct, there would be no way to form SMNSs via accretion from a companion in a LMXB.

Both Cook, Shapiro and Teukolsky (1994b) and Possenti et al.(1999) halted their computations when the mass shedding rotation rate is reached, but there is nothing magic about this moment. Instead, Popham and Narayan (1991) and Paczynski (1991) argued that accretion continues unimpeded, in Newtonian stars plus disks configurations, with stars remaining close to the breakup angular speed, while mass and total angular momentum increase. Other reasonable possibilities may contribute to prolong mass accretion: the reduction of angular momentum through gravitational wave losses (propitiated by the 
growth of a small stellar eccentricity) or the setup of a spiral shock wave reducing the angular momentum of incoming disk material, with the outward transport of angular momentum. In any case, several avenues are possible which would keep the neutron star marginally inside the mass shedding limit. For this reason, it seems nearly certain that the intermediate EoSs $(\mathrm{C}, \mathrm{M}, \mathrm{UT}, \mathrm{FPS})$ which are only $\approx 0.1 \mathrm{M}_{\odot}$ away from being supramassive, will reach this stage as mass accretion continues.

Since all soft and intermediate equations of state only require $\approx 0.5 \mathrm{M}_{\odot}$ to become supramassive, their companion star may be any star with mass $\lesssim 1 \mathrm{M}_{\odot}$, exactly as discussed in the normal recycling model for MSPs. EoSs AU and UT, instead, require in total $\approx 1-1.1 \mathrm{M}_{\odot}$ to become supramassive. At first sight, this requirement might seem unsurmountable: thermal stability in the mass exchange process through Roche lobe overflow (Webbink et al.1983) requires that the companion of the NS has a mass below $5 / 6$ of the neutron star's, i.e., $1.17 \mathrm{M}_{\odot}$ for an initial NS mass of $1.4 \mathrm{M}_{\odot}$. Since the smallest He-core that may be left behind is that of a star which took all Hubble time to evolve off the main sequence, $0.16 \mathrm{M}_{\odot}$, this leaves a maximum transferable mass of $1.01 \mathrm{M}_{\odot}$, less than required for either AU or UT. However, this is incorrect: the famous requirement of 5/6ths only occurs because Webbink et al.(1983) considered Paczynski's (1967) approximation for the Roche lobe radius: using instead Eggleton's (1983) formula, this requirement disappears. Webbink et al.(1983, Eq. 15) show that the mass transfer rate is $\dot{M}_{1} \propto-1 /\left(d \ln R_{L} / d \ln M_{1}\right)$ where $R_{L}$ is the Roche lobe radius, and they argue that thermal stability in the process requires $X \equiv d \ln R_{L} / d \ln M_{1}>0$. Using Eggleton's formula $R_{L} / a=0.49 /\left(0.6+\ln \left(1+q^{1 / 3}\right) / q^{2 / 3}\right)$ where $a$ is the distance between the two stars and $q \equiv M_{1} / M_{2}$ is the mass ratio, we find, under the hypothesis of conservative mass transfer,

$$
X=\frac{(1+q)\left(2\left(1+q^{1 / 3}\right) \ln \left(1+q^{1 / 3}\right)-q^{1 / 3}\right)}{\left(1+q^{1 / 3}\right)\left(1.8 q^{2 / 3}+3 \ln \left(1+q^{1 / 3}\right)\right)}>0
$$

for every $q$ ! Also, dynamical stability exists provided the donor mass is $<2 \mathrm{M}_{\odot}$ (Rappaport 
et al., 1995). So we may consider as a possible companion for neutron stars with EoSs AU and UT, sub/giants of mass $\lesssim 2 \mathrm{M}_{\odot}$, where mass transfer is pushed forth by donor radius expansion, in complete analogy with the model of Webbink et al., 1983, except for donor mass. From Fig. 8b of Verbunt (1993), we see that a giant or subgiant of nearly solar metallicity, of, say, $1.7 \mathrm{M}_{\odot}$ manages to transfer at sub-Eddington rates $\approx 1.4 \mathrm{M}_{\odot}$ to the neutron star, provided mass transfer begins when the giant core is $\approx 0.2 \mathrm{M}_{\odot}$; mass transfer will then leave behind a small $\left(\approx 0.3 \mathrm{M}_{\odot}\right)$, nearly inert He nucleus, with final period in the range of $0.3 \mathrm{~d}$. We thus see that these systems provide attractive progenitors for supramassive neutron stars, even in the case in which the applicable EoS is either AU or UT, provided of course mass accretion is close to conservative, an implicit assumption we made throughout, and that mass can be accreted in sufficient quantities.

Recent studies of binary pulsar masses (Thorsett and Chakrabarty 1999) seem to argue against significant mass accretion, but it should be noticed that, by investigating millisecond pulsars with periods exceeding $\approx 2 \mathrm{~ms}$, the authors are investigating objects for which we know a priori that little mass need have been accreted, since their periods are long compared with SMNSs'. We may expect different results when pulsars are chosen otherwise: a recent redetermination of the mass of Cyg X-2 finds $M=(1.8 \pm 0.2) M_{\odot}$ (Orosz and Kuulkers 1999), departing from the narrow range of Thorsett and Chakrabarty.

The lowest magnetic field for the formation of supramassive neutron stars may be lower than the empyrical value $\left(\approx 2 \times 10^{8} \mathrm{G}\right)$ mentioned above (Possenti et al. (1999) because when mass accretion from the companion begins to taper off, or alternatively if mass accretion is intermittent, the Alfvén radius (which scales as $\dot{M}^{-2 / 7}$ ) may expand further than the corotation radius: the neutron star then goes through a new propeller phase which slows its rotation. The overall effect is not large so that we shall consider in the following a maximum magnetic field $q \times 10^{8} \mathrm{G}$, with $q \lesssim 1$. 


\section{Further evolution}

Supramassive neutron stars are unstable to collapse to a black hole when angular momentum losses reduce the initial angular momentum to about half of the initial value; furthermore, these stars are peculiar in that evolution at constant baryon number, but decreasing total angular momentum, makes them spin up, rather than down; all of this is especially evident in Fig. 7-10-13-16 of Salgado et al., 1994. Magnetic dipole losses cause a net torque which spins down the neutron star in a time (Vietri and Stella 1998) $t_{s d}=5 \times 10^{9} \operatorname{yr}\left(10^{8} \mathrm{G} / \mathrm{B}\right)^{2}$. This time-scale is not strongly dependent upon EoS, but depends strongly upon whether the model is only marginally supramassive, or close to the absolute maximum mass (rotating or not) for the given EoS, so that it may be considerably shorter under many circumstances. Thus, a time $t_{s d}$ after becoming supramassive, the neutron star will collapse to a black hole. This time is reasonably long when compared with typical mass accretion time-scales, which, as discussed above, are typically determined by sub/giant nuclear evolution timescales. Thus mass transfer will have long since ceased, and the immediate SMNS surroundings will be reasonably baryon free. The companion star, in the meantime, will have settled down as a low-luminosity, low-mass white dwarf, which is not expected to pollute the environment either. Furthermore, we can gauge the baryon-cleanliness of the SMNS surroundings at large if we assume that MSPs are born through the same chain of events, except less extreme, for then we know the Galactic distribution of MSPs. These are often located well outside the Galactic disk, within an ISM with typical densities well in defect of $n=1 \mathrm{~cm}^{-3}$, which makes the total baryon mass within, say, $0.1 \mathrm{pc}$, less than $10^{-5} \mathrm{M}_{\odot}$, more than enough to guarantee contact with the fireball model. We thus see that also this version of the formation scenario guarantees a baryon clean environment, exactly like the different scenario of Paper I.

The situation is clean even in the case in which the collapse occurs while mass transfer 
is still taking place. The total amount of baryons in the accretion disk is negligible: the disk crossing time is of order of $\approx 1$ month, which, with mass transfer rates $10^{-9}-10^{-8} M_{\odot} y r^{-1}$, corresponds to much less than the maximum contamination value. The total amount of outlying mass from a wind is also rather small: for $\dot{M}_{w} \approx 10^{-9} M_{\odot} y r^{-1}$ and $v_{w} \approx 30 k m s^{-1}$, the total mass within, say, $0.1 p c$ is $3 \times 10^{-6} M_{\odot}$, again negligible. The highly relativistic ejecta and $\gamma$ rays from the burst will hit the companion and form a shock way inside the star's photosphere, so that local dissipation of the ejecta kinetic energy will lead to the companion's inflating on the (long!) Kelvin-Helmholtz time-scale, and the non-thermal afterglow emission will not be contaminated by the re-radiated thermal component.

The mechanism for the energy release is the same as discussed in paper I: once the neutron star is destabilized, the innermost regions will collapse promptly to a black hole, while the equatorial matter, which is close to centrifugal equilibrium, will just contract a little bit and begin orbiting the newly formed black hole. A necessary condition which needs to be met is that this equatorial material lies outside the innermost stable orbit. This can be checked from Table I of Cook, Shapiro and Teukolsky (1994b) who show that neutron stars which have reached the mass shedding regime have equatorial radii larger than the innermost stable orbit (see their column $j$ ), independent of EoS. In paper I, we estimated the amount of matter left behind as $\approx 0.1 \mathrm{M}_{\odot}$; this configuration is identical to the one hypothesized in most current models (Mèszàros 1999), and the debris torus is massive enough to power any burst, especially in the presence of a moderate amount of beaming.

We now discuss the rate at which spun-up SMNSs collapse to black holes. Since the timescales involved are a fair fraction of the age of the Universe, and since star formation evolves strongly in the recent past (Madau et al., 1996), we have to consider cosmological evolution of the population. However, from Fig.1 of White and Ghosh (1998), it can be seen that the population of MSPs is roughly constant (within the accuracy of the present, order 
of magnitude estimates) over the redshift range $0<z \lesssim 1$ for most assumptions. There are currently an estimated $5 \times 10^{4}$ MSPs in the disk of the Galaxy (Lorimer 1995, Phinney and Kulkarni 1994); assuming that there are as many systems in the bulge, that a fraction $\beta$ of these are SMNSs, and that the typical timescale for collapse to black hole is given by $t_{s d}$, the expected rate of collapses in the Milky Way is $r=10^{5} \beta / t_{s d}=\beta /\left(5 \times 10^{4} \mathrm{yr}\right)$, which is to be compared with the inferred rate of GRBs, 1 every $3 \times 10^{7} \mathrm{yr}$ in an $L_{\star}$ galaxy like the Milky Way. Scaling to $\beta=0.05$, a value intermediate between the extremes of the simulations of Possenti et al., we find that the two rates agree for a beaming fraction $\delta \Omega / 4 \pi \approx 0.2(\beta / 0.05) ;$ this is consistent with the idea that these explosions do not require extreme beaming fractions, since the explosion need not wade its way through a massive stellar envelope, but immediately breaks free into a baryon clean environment.

This model makes an easily testable prediction, because the location of bursts inside their host galaxies is the same as that of LMXBs, which are distributed at distances from the Galactic plane $\bar{z} \approx 1 \mathrm{kpc}$, most likely arising from kick velocities at the time of neutron star formation (van Paradijs and White 1995). A similar $z$ distribution is observed for MSPs, $\bar{z} \gtrsim 0.7 \mathrm{kpc}$, and moderate transverse speeds. Thus we would expect GRBs to cluster around galactic disks (contrary to the binary pulsar merger model, where at least some $50 \%$ of all GRBs should be uncorrelated with the original birth galaxies), but should not correlate with star forming regions (except for SMNSs which form directly during a SN event, as discussed in paper I), contrary to all scenarios involving massive stars. Also, the redshift distribution of GRBs within this model should be flatter than the star formation distribution (again contrary to hypernovae), because the redshift distribution of the MSP population is rather flat (White and Ghosh 1998).

We acknowledge helpful discussions with G. Ghisellini, A. Possenti and L. Burderi. 


\section{REFERENCES}

Arons, J., 1993, ApJ, 408, 160.

Burderi, L., Possenti, A., Colpi, M., Di Salvo, T., D’Amico, N., 1999, ApJ, 519, 285.

Camilo, F., Nice, D.J., Taylor, J.H., 1996, ApJ, 461, 812.

Cook, G.B., Shapiro, S.L., Teukolsky, S.A., 1994a, ApJ, 424, 823.

Cook, G.B., Shapiro, S.L., Teukolsky, S.A., 1994b, ApJ, 423, L117.

Eggleton, P.P., 1983, ApJ, 268, 368.

Ghosh, P., Lamb, F.K., 1978, ApJ, 223, L83.

Ghosh, P., Lamb, F.K., 1992, in X-ray binaries and formation of of binary and millisecond pulsars, ed. E. van den Heuvel and S. Rappaport, Dordrecht, Kluwer (1992), 487.

Illarionov, A., Sunyaev, R., 1975, A\&A, 39, 185.

Lorimer, D.R., 1995, MMNRAS, 274, L300.

Madau, P., Ferguson, H.C., Dickinson, M.E., Giavalisco, M., Steidel, C.C., Fruchter, A., 1996, MNRAS, 283, 1388.

Mèszàros, P., 1999, astro-ph. 9904038.

Miller, M.C., Lamb, F.K., Psaltis, D., 1998, ApJ, 508, 791.

Orosz, J.A., Kuulkers, E., 1999, MNRAS, 305, 132.

Paczynski, B., 1967, Acta Astron., 17, 287.

Paczynski, B., 1991, ApJ, 370, 593.

Phinney, E.S., Kulkarni, S., 1994, ARA\&A, 32, 591.

Popham, R., Narayan, R., 1991, ApJ, 370, 614. 
Possenti, A., Colpi, M., Geppert, U., Burderi, L., D'Amico, N., 1999, ApJS, in press, astro-ph 9907231.

Psaltis, D., Chakrabarty, D., 1999, ApJ, 521, 332.

Rappaport, S., Podsiadlowski, P., Joss, P.C., Di Stefano, R., Han, Z., 1995, MNRAS, 273, 731.

Rees, M.J., Mészáros, P., 1992, MNRAS, 258, 41P.

Ruderman, M., Zhu, T., Chen, K., 1998, ApJ, 492, 267.

Salgado, M., Bonazzola, S., Gourgoulhon, E., Haensel, P., 1994, A\&A, 291, 155.

Stella, L., Vietri, M., 1999, Phys. Rev. Lett., 82, 17.

Stella, L., Vietri, M., Morsink, S., 1999, ApJ, in press, astro-ph 9907346.

Thorsett, S.E., Chakrabarty, D., ApJ, 512, 288.

van der Klis, M., 1998, in Proccedings of the 3rd William Fairbank Meeting, to appear, astro-ph 9812395.

van Paradijs, J., White, N., 1995, ApJ, 447, L33.

Verbunt, F., 1993, ARA\&A, 31, 93

Vietri, M., Stella, L., 1998, ApJ, 507, L45.

Webbink, R.F., Rappaport, S., Savonije, G.J., 1983, ApJ, 270, 678.

White, N.E., Ghosh, P., 1998, ApJ, 504, L31. 
Accretion from Keplerian disk onto a $1.4 \mathrm{M}_{\odot}$ neutron star

\begin{tabular}{|c|c|c|c|c|c|c|c|}
\hline $\mathrm{EoS}^{a}$ & $M_{f} / M_{\odot}^{b}$ & $M_{i} / M_{\odot}^{c}$ & $\triangle M / M_{\odot}^{d}$ & $P_{f}^{e}$ & $R_{i}^{f}$ & $h_{i}^{g}$ & $M_{s m} / M_{\odot}^{h}$ \\
\hline $\mathrm{A}$ & 1.77 & 1.57 & 0.428 & 0.604 & 9.59 & 2.82 & 1.66 \\
$\mathrm{C}$ & 1.74 & 1.54 & 0.389 & 0.894 & 12.1 & 0.27 & 1.86 \\
$\mathrm{D}$ & 1.76 & 1.56 & 0.405 & 0.730 & 10.7 & 1.70 & 1.65 \\
$\mathrm{E}$ & 1.76 & 1.57 & 0.414 & 0.656 & 10.0 & 2.38 & 1.75 \\
$\mathrm{~F}$ & 1.52 & 1.59 & 0.172 & 0.715 & 9.21 & 3.20 & 1.46 \\
$\mathrm{~L}$ & 1.80 & 1.52 & 0.443 & 1.250 & 15.0 & 0.00 & 2.70 \\
$\mathrm{M}$ & 1.74 & 1.50 & 0.367 & 1.490 & 16.7 & 0.00 & 1.80 \\
$\mathrm{~N}{ }^{\star}$ & 1.84 & 1.53 & 0.484 & 1.080 & 13.6 & 0.00 & 2.64 \\
$\mathrm{KC}$ & 1.74 & 1.55 & 0.385 & 0.888 & 12.1 & 0.31 & 1.49 \\
$\mathrm{AU}$ & 1.79 & 1.58 & 0.446 & 0.701 & 10.4 & 2.01 & 2.13 \\
$\mathrm{UU}$ & 1.78 & 1.56 & 0.436 & 0.784 & 11.2 & 1.26 & 2.20 \\
$\mathrm{UT}$ & 1.78 & 1.57 & 0.429 & 0.754 & 10.9 & 1.52 & 1.84 \\
$\mathrm{FPS}$ & 1.76 & 1.56 & 0.416 & 0.747 & 10.9 & 1.56 & 1.80 \\
\hline
\end{tabular}

Table 1: From Cook, Shapiro and Teukolsky, 1994b. ${ }^{a}$ Equation of state; ${ }^{b}$ final total massenergy: ${ }^{c}$ initial rest mass; ${ }^{d}$ accreted rest mass; ${ }^{e}$ rotation period in $m s ;{ }^{f}$ initial circumferential radius in $\mathrm{km} ;{ }^{g}$ initial circumferential height of corotating marginally stable orbit in $\mathrm{km} ;{ }^{h}$ maximum static total mass-energy for EoS. 